\section{Small bone marrow adipocytes predict poor prognosis in acute myeloid leukemia}

Being overweight or obese is associated with an increased risk of developing leukemia and relapse, ${ }^{1,2}$ suggesting that adipose tissue plays an important role in this disease. It has been demonstrated that adipocytes in the bone marrow provide a pro-tumoral microenvironment during the initial stage of leukemia. ${ }^{3}$ However, these adipocytes are rapidly reduced with the extensive proliferation of leukemic blasts in the bone marrow. There are limited reports regarding the regulation of the residual adipocytes by leukemic cells and their role in disease progression. Generally, adipocyte morphology may indicate pathological function in vivo. For example, large adipocytes occupy the bone marrow cavity in aplastic anemia and negatively regulate hematopoiesis. ${ }^{4}$ In the case of solid tumors, adipocyte size is dramatically reduced with the progression of cancer cell aggressiveness. ${ }^{5,6} \mathrm{We}$ hypothesize that the residual marrow adipocytes are regulated to acquire special pathological characteristics in response to leukemic development.

To explore the morphological characteristics of bone marrow adipocytes in patients with acute myeloid leukemia (AML), we retrospectively analyzed the size of marrow adipocytes in bone marrow sections from 70 patients with primary AML and 70 controls. The median ages of the primary AML patients and controls were 55 years (range, 18-82 years) and 52 years (range, 18-86 years), respectively. The gender ratios (male/female) in these two groups were $32 / 38$ and $37 / 33$, respectively. Among the 70 AML patients, 32 patients were classified as belonging to the remission group because they achieved complete remission after induction chemotherapy, and 38 patients were classified as the refractory group according to the criteria of Schmid C. 7 There were no significant differences between these two groups regarding the patients' basic characteristics, peripheral blood white cell count or bone marrow blast percentage (Online Supplementary Table S1). Adipocytes, confirmed by immmunhistochemistry to be positive for Perilipin 1 staining, were fewer and smaller in the bone marrow sections from AML patients than from the controls (Online Supplementary Figure S1A). Furthermore, quantitative analysis showed that both the adipocyte diameter and adipocyte area in the AML patients were significantly smaller (average $38.3 \mu \mathrm{m}$ and $560 \mu \mathrm{m}^{2}$ ) when compared with those of the controls (average 51.2 $\mu \mathrm{m}$ and 1137 $\mu^{2} ; P<0.001$; Online Supplementary Figure $\left.S 1 B, C\right)$. Subsequently, the linear analysis results showed that neither adipocyte diameter nor adipocyte area was correlated with bone marrow blast percentage $(P=0.21 ; P=0.245$; Online Supplementary Figure $S 1 D, E)$, indicating that adipocyte size change in the AML patients was not caused by a lack of space due to extensive invasion of leukemic cells.
A

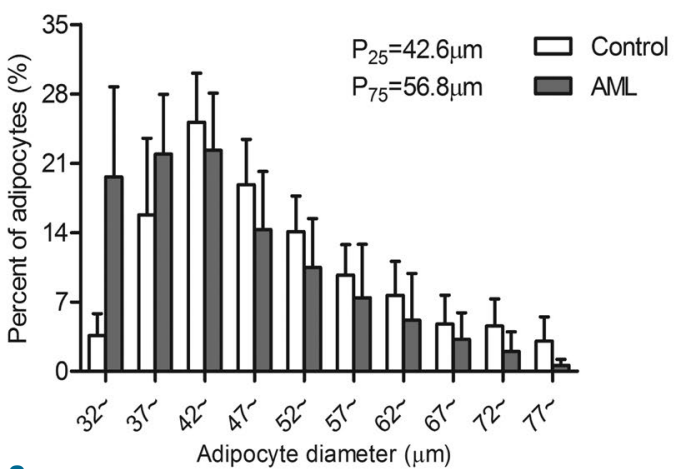

C

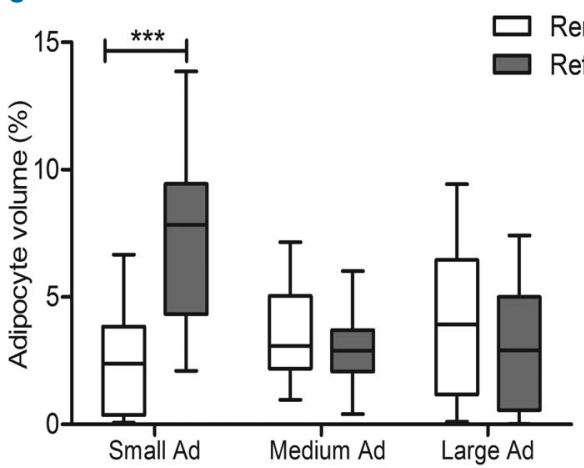

B

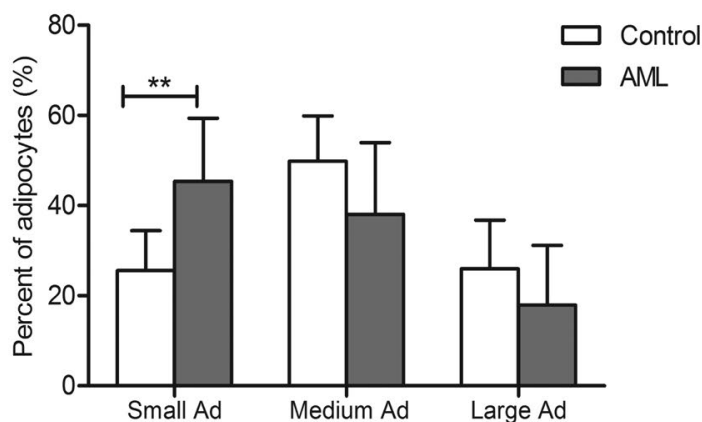

D

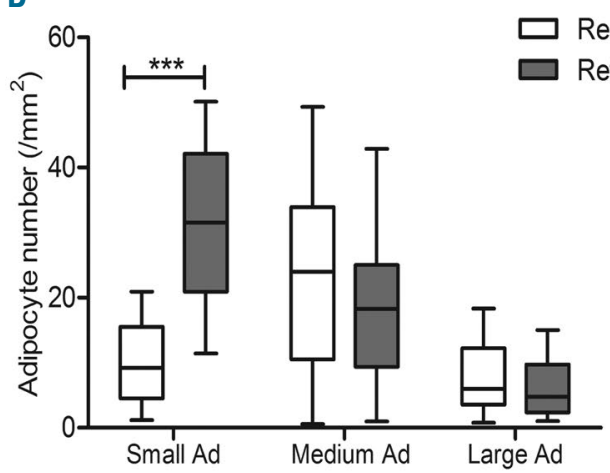

Figure 1. Small adipocytes in the bone marrow of patients with acute myeloid leukemia. (A) Frequency distribution of adipocyte diameter in the controls ( $\mathrm{n}=70$ ) and AML patients $(n=70)$. The $25^{\text {th }}$ percentile (P25) and the $75^{\text {th }}$ percentile (P75) of adipocyte diameter in the controls were $42.6 \mu \mathrm{m}$ and $56.8 \mu \mathrm{m}$, respectively. (B) The proportion of adipocytes with different sizes classified by adipocyte diameter determined by the Mann-Whitney $U$ test in all AML patients ( $=70$ ) and the controls $(n=70) . * * P<0.01$. (C and D) Box-plots showing the volume $(C)$ and number $(D)$ of small, medium-sized and large adipocytes in remission $(n=32)$ and refractory patients $(\mathrm{n}=38)$; Mann-Whitney $U$ test. $* * * P<0.001$. 
A

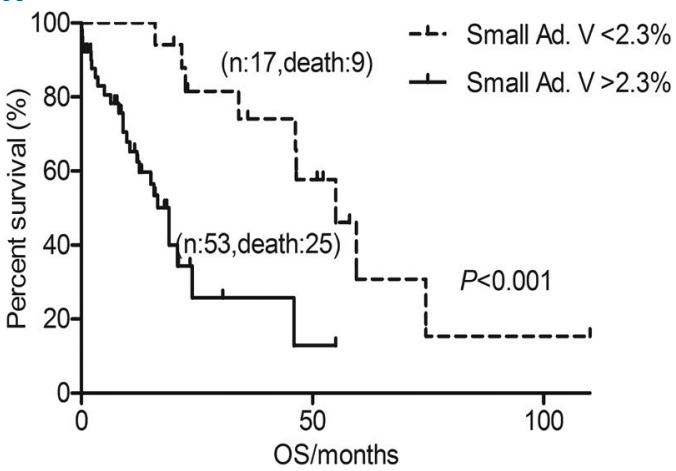

C

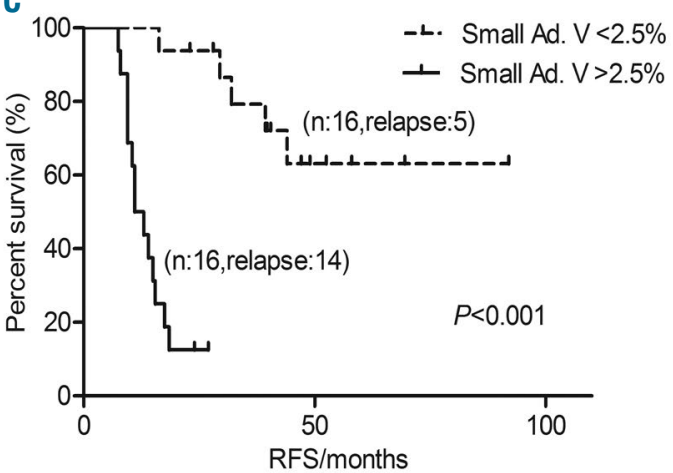

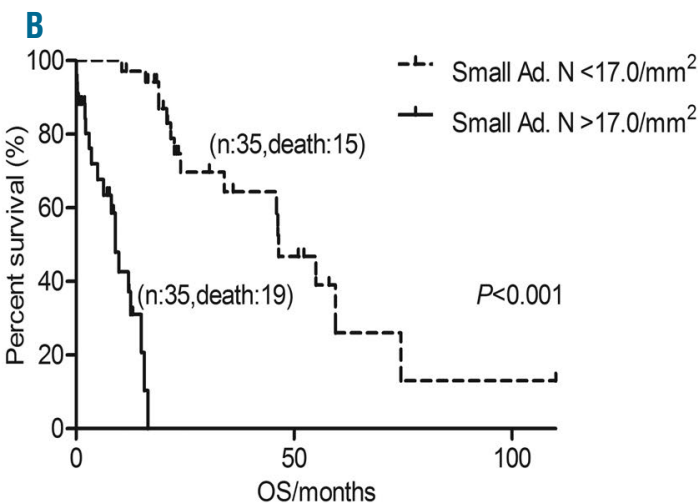

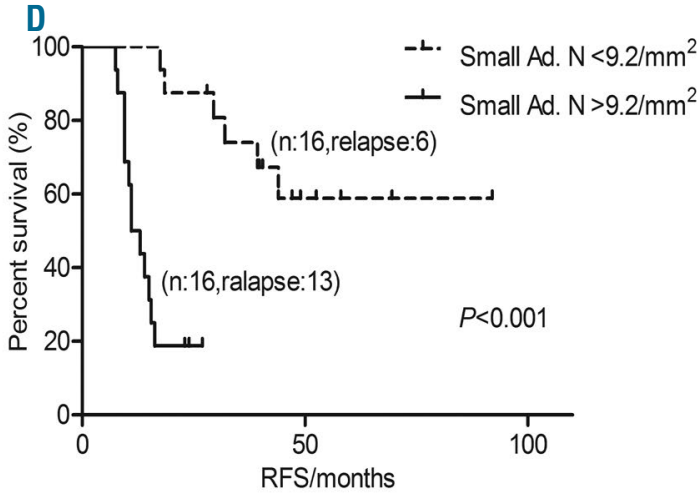

Figure 2. Impact of small adipocytes on the outcome of patients with acute myeloid leukemia. (A and B) Kaplan-Meier curves of less small adipocytes (small adipocyte volume $<2.3 \%$ or small adipocyte number $<17.0 / \mathrm{mm}^{2}$ ) and more small adipocytes (small adipocyte volume $>2.3 \%$ or small adipocyte number $>17.0 / \mathrm{mm}^{2}$ ) for the overall survival (OS) of AML patients. $P<0.001$. (C and D) Kaplan-Meier curves of less small adipocytes (small adipocyte volume $<2.5 \%$ or small adipocyte number $<9.2 / \mathrm{mm}^{2}$ ) and more small adipocytes (small adipocyte volume $>2.5 \%$ or small adipocyte number $>9.2 / \mathrm{mm}^{2}$ ) for the relapse-free survival (RFS) of AML patients. $P<0.001$. Optimal cut-off values for less and more small adipocytes in this study were determined by Cox analysis for the OS or RFS of AML patients dividing the material by the quartiles (Online Supplementary Table S4).

The marrow adipocytes were divided into three subpopulations based on the values of the $25^{\text {th }}$ and $75^{\text {th }}$ percentiles of their diameter or area in the controls. ${ }^{8}$ Adipocytes with a diameter less than $42.6 \mu \mathrm{m}$ were regarded as small adipocytes, while those with a diameter greater than $56.8 \mu \mathrm{m}$ were defined as large adipocytes. Adipocytes with an intermediate diameter were regarded as medium-sized adipocytes (Figure 1A). Only the proportion of small adipocytes was higher in the AML patients than in the controls $(43.9 \% \pm 19.3 \%$ versus $25 \% \pm 8.9 \%$, respectively; $P=0.008$ ) (Figure $1 \mathrm{~B}$ ), whereas neither medium-sized adipocytes nor large adipocytes exhibited significant differences between these two groups. Similar results were also obtained using adipocyte area as a discriminatory feature (Online Supplementary Figure S2A,B). Furthermore, we found that the volume and number of small adipocytes in the refractory subjects $(7.0 \%$ and $31.6 / \mathrm{mm}^{2}$ ) were significantly greater than those in the remission subjects $\left(2.4 \%\right.$ and $\left.9.8 / \mathrm{mm}^{2}, P<0.001\right)$ (Figure $1 C, D)$. However, no differences were found for mediumsized or large adipocytes between these two groups. These results led us to analyze the relationship between small adipocytes and AML patients' survival.

To determine whether small adipocytes are risk factors for the survival of AML patients, we used Cox regression analyses. Univariate Cox regression identified age and the volume and number of small adipocytes as risk factors for overall survival and relapse-free survival of AML patients (Online Supplementary Table S2). However, a multivariate
Cox regression showed that only the volume and number of small adipocytes were associated with a high risk of relapse and death in the AML patients (Online Supplementary Table S3). The cut-off values for KaplanMeier survival analysis were decided to be the quartiles of the small adipocyte volume and number in Cox analyses, ${ }^{9}$ and the values that had the highest hazard ratio were used as the optimal cut-off values (Online Supplementary Table S4). Patients with more small adipocytes (small adipocyte volume $\geq 2.3 \%$ or small adipocyte number $\geq 17.0 / \mathrm{mm}^{2}$ ) had a shorter overall survival compared with the others (average overall survival 11.5 months versus 44.1 months, or 5.9 months versus 33.0 months, respectively, $P<0.001$ ) (Figure 2A,B). Regarding the AML remission subjects, patients with more small adipocytes (small adipocyte volume $\geq 2.5 \%$ or small adipocyte number $\geq 9.2 / \mathrm{mm}^{2}$ ) had a shorter relapse-free survival compared with the others (average 13.8 months versus 44.3 months, or 14.0 months versus 44.1 months, respectively, $P<0.001$ ) (Figure 2C,D). These results suggest that small adipocytes might be a risk factor for poor prognosis of AML patients.

The possible reasons for the generation of small adipocytes in AML were explored. Recent studies showed that adipocytes around tumor cells became small and involved in tumor metastasis and growth. ${ }^{10,11} \mathrm{We}$, therefore, evaluated the effect of leukemic cells on marrow adipocytes, as leukemic cells diffuse in the bone marrow cavity and are in close contact with the marrow adipocytes. We co-cultured AML cell lines and primary 
A $\mathrm{Ad}+\mathrm{HC}$ Ad+THP-1 $\mathrm{Ad}+\mathrm{K} 562$

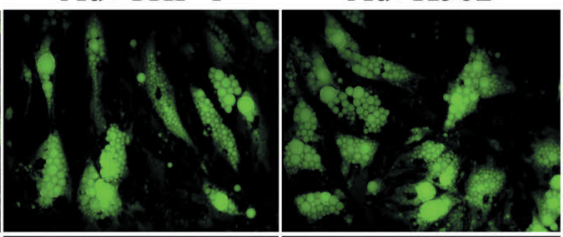

Ad+HL-60

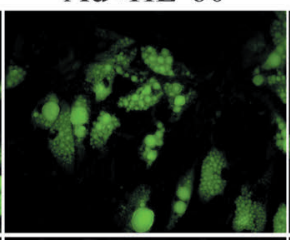

$\mathrm{Ad}+\mathrm{LC}$
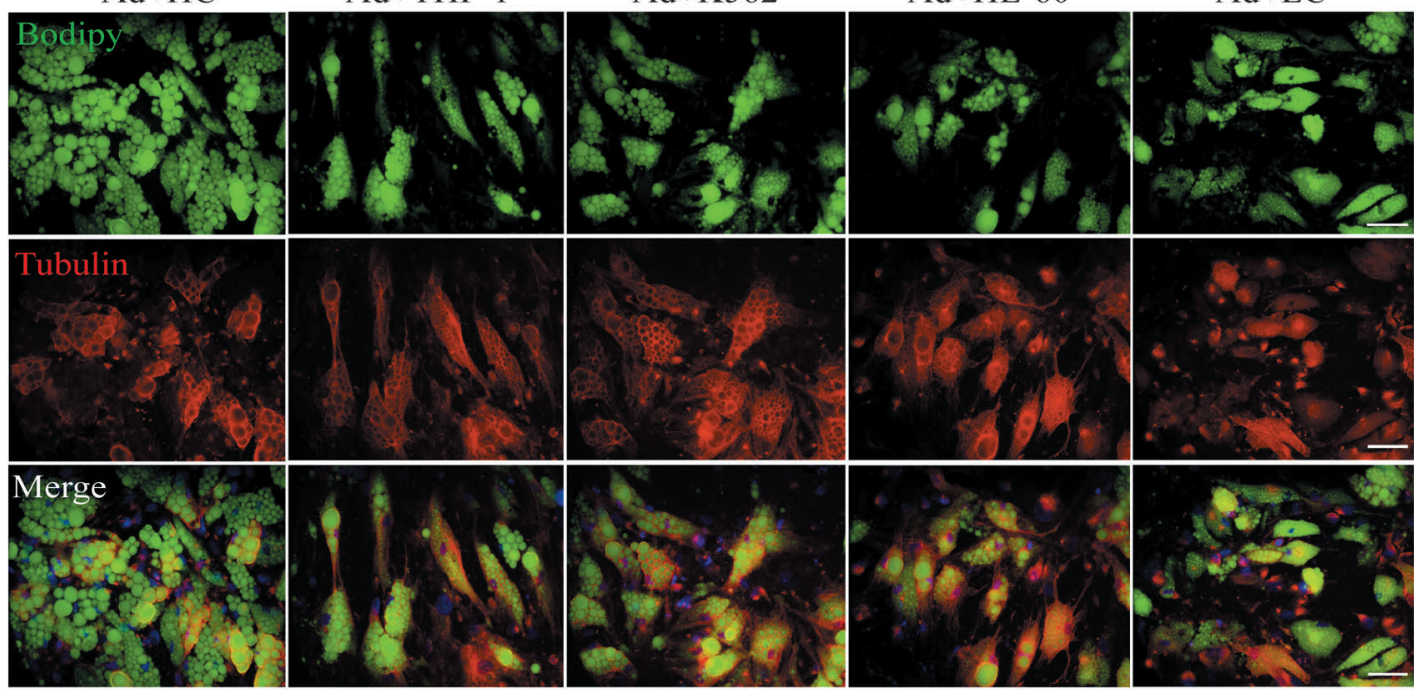

B

C
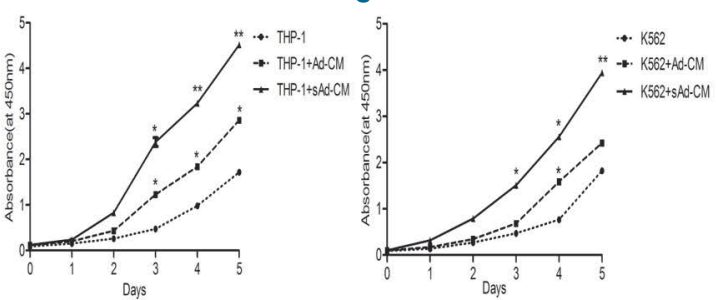

D

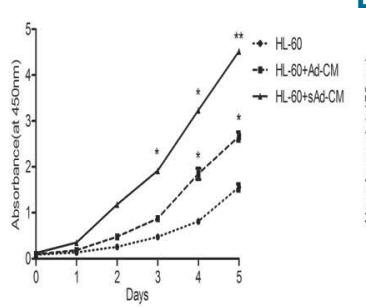

E

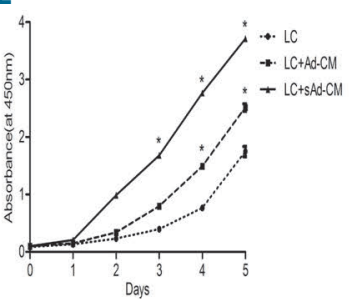

Figure 3. Leukemic cells induce the generation of small adipocytes. (A) Bone marrow mesenchymal stem cells-derived adipocytes co-cultured with primary hematopoietic cells (Ad+HC), the THP-1 cell line (Ad+THP-1), K562 cell line (Ad+K562), HL-60 cell line (Ad+HL-60) and primary AML blasts (Ad+LC) for 5 days. Adipocytes were detected by Bodipy (green) and Tubulin (red) staining. Nuclei were labeled with DAPI. The Ad+HC group served as the control. Images were acquired using a magnification of $\times 60$ by confocal microscopy. The scale bar represents $25 \mu \mathrm{m}$. (B) CCK 8 detection of the proliferation of THP-1, K562, HL- 60 cell lines and primary AML blasts (LC) cultured with the conditioned medium of small adipocytes (sAd-CM) or the conditioned medium of normal adipocytes (Ad$\mathrm{CM})$. Cell lines and LC cultured alone were used as the control. Values shown are the mean \pm standard error of mean. Statistical differences between the Ad$\mathrm{CM}$ and control groups and between the SAd-CM and Ad-CM groups were detected by the Mann-Whitney $\mathrm{U}$ test. $* P<0.05, * * P<0.01$.

AML blasts with bone marrow stromal cell-derived adipocytes through a transwell system. According to Bodipy staining results, the adipocyte lipid-droplets decreased significantly or were lost (Figure 3A), which might be consistent with increased lipolytic gene expression (hormone-sensitive triglyceride lipase, HSL and adipose triglyceride lipase, ATGL) (Online Supplementary Figure $S 3 B$ ). Meanwhile, the adipocyte sizes were dramatically reduced, as demonstrated by tubulin staining, and this result was further confirmed through quantitative analysis using Image-Pro Plus 5.1 (Online Supplementary Figure S3A). Since leukemic cells could induce the formation of small adipocytes from large, mature adipocytes through the co-culture system, it was not possible to test the effect of large adipocytes on leukemic cell proliferation. We, therefore, cultured AML cells in the conditioned medium obtained from mature adipocytes and small adipocytes. Interestingly, with the reduction of free fatty acids (FFA) in the culture system (Online Supplementary Figure $S 3 C$ ), the proliferation of AML cells cultured in small adipocyte conditioned medium was faster than that from mature adipocyte conditioned medium (Figure 3BE). These results suggest that leukemic cells may regulate the transition of mature adipocytes to small adipocytes, and in turn, small adipocytes may promote leukemic cell proliferation. Finally, we retrospectively analyzed the cor- relation between small adipocyte content (the percentage of small adipocytes in all adipocytes) and the percentage of bone marrow blasts. The positive correlation between them might shed further light on the effect of small adipocytes on AML cells in vivo $(P=0.0048, \mathrm{R}=0.33$ (Online Supplementary Figure S3D).

In this study, we found that small adipocytes were increased in AML patients after comparing the proportion of adipocytes of different sizes between AML patients and controls. Interestingly, large and medium-sized adipocytes were reduced in AML patients; however, these changes were not statistically significant, indicating that the increase in small adipocytes might not entirely depend on the decrease in large and medium-sized adipocytes. Considering risk factors for AML patients' survival, patients' age was included in our study, as it has been well established as the strongest patient-related prognostic factor in AML. ${ }^{12}$ However, we found that it was the volume and number of small adipocytes, and not age, that were significantly correlated with the patients' prognosis according to multivariable Cox regression analyses. It may be relevant that there was no difference between the age of remission cases and refractory cases.

Our results indicate that leukemic cells contributed to the generation of small adipocytes in vitro. Lipolysis plays an important role in morphological change of adipocytes, 
which is consistent with a previous report that smaller adipocyte size was linked to higher metabolic activity. ${ }^{13}$ Additionally, it has been reported that tumor cells release inflammatory factors to reduce adipocyte size. ${ }^{14}$ We also found that growth differentiation factor 15, a member of the transforming growth factor-beta family, secreted by leukemic cells may be involved in the generation of small adipocytes (data not shown). FFA was released by adipocytes during lipolysis. It has been reported that FFA derived from adipocyte lipolysis is transported by FABP 4 to AML cells. ${ }^{3}$ In our study, the decreased FFA in the adipocytes co-cultured with leukemic cells may be attributed to the transportation of FFA from adipocytes to leukemic cells, which might promote leukemic cell proliferation. To some extent this would explain the adverse effects of small adipocytes on the prognosis of patients with AML.

In summary, we have highlighted the presence of small bone marrow adipocytes and demonstrate that only these adipocytes, and not the total adipocytes, are correlated with a poor prognosis for AML patients. To our knowledge, no other human studies have investigated the correlation between morphological changes of bone marrow adipocytes and the prognosis of patients with AML. Our results strongly suggest that small adipocytes in the bone marrow may serve as an adverse prognostic factor and provide some evidence to support the concept that disrupting AML-adipocyte interactions could be a targeted therapeutic approach for AML.

Wei Lu, Wei Weng, ${ }^{2}$ Qi Zhu, ${ }^{3}$ Yuanmei Zhai, ${ }^{2}$ Yun Wan, ${ }^{1,4}$ Haiyan Liu, ${ }^{1}$ Shaoxin Yang, ${ }^{1}$ Yehua Yu, ${ }^{3}$ Yanyu Wei ${ }^{1}$ and Jun Shi'

'Department of Hematology, Shanghai Jiao Tong University Affiliated Sixth People's Hospital, Shanghai; ${ }^{2}$ Department of Hematology, Shanghai Jiao Tong University School of Medicine Affiliated Tongren Hospital, Shanghai; ${ }^{3}$ Department of Hematology, Shanghai Jiao Tong University School of Medicine Affiliated Ninth People's Hospital, Shanghai and ${ }^{4}$ Department of Oncology, Kunshan Hospital of Traditional Chinese Medicine, Kunshan, China

WL and WW contributed equally to this work.

Funding: this study was supported by the National Natural Science Foundation of China (grant n. 81570135), National Science and Technology Major Equipment Projects of China (grant n. 2013YQ03065109) and Science and Technology Commission of Shanghai Municipality (grant n.14142200700).

Correspondence: junshi@sjtu.edu.cn

\section{doi:10.3324/haematol.2017.173492}

Information on authorship, contributions, and financial \& other disclosures was provided by the authors and is available with the online version of this article at www. haematologica.org.

\section{References}

1. Larsson SC, Wolk A. Overweight and obesity and incidence of leukemia: a meta-analysis of cohort studies. Int J Cancer. 2008;122(6):1418-1421

2. Orgel E, Tucci J, Alhushki W, et al. Obesity is associated with residual leukemia following induction therapy for childhood B-precursor acute lymphoblastic leukemia. Blood. 2014;124(26):3932-3938.

3. Shafat MS, Oellerich T, Mohr S, et al. Leukemic blasts program bone marrow adipocytes to generate a pro-tumoral microenvironment. Blood. 2017;129(10):1320-1332.

4. Naveiras O, Nardi V, Wenzel PL, Hauschka PV, Fahey F, Daley GQ. Bone-marrow adipocytes as negative regulators of the haematopoietic microenvironment. Nature. 2009:460(7252):259-263.

5. Tan J, Buache E, Chenard MP, Dali-Youcef N, Rio MC. Adipocyte is a non-trivial, dynamic partner of breast cancer cells. Int J Dev Biol. 2011;55(7-9):851-859.

6. Tokuda Y, Satoh Y, Fujiyama C, Toda S, Sugihara H, Masaki Z. Prostate cancer cell growth is modulated by adipocyte-cancer cell interaction. BJU Int. 2003;91(7):716-720.

7. Bennett JM, Catovsky D, Daniel MT, et al. Proposals for the classification of the acute leukaemias. French-American-British (FAB) cooperative group. Br J Haematol. 1976;33(4):451-458.

8. Langouche L, Perre SV, Thiessen S, et al. Alterations in adipose tissue during critical illness: an adaptive and protective response? Am J Respir Crit Care Med. 2010;182(4):507-516.

9. Norén-Nyström U, Roos G, Bergh A, et al. Bone marrow fibrosis in childhood acute lymphoblastic leukemia correlates to biological factors, treatment response and outcome. Leukemia. 2008;22(3):504510 .

10. Herroon MK, Rajagurubandara E, Hardaway AL, et al. Bone marrow adipocytes promote tumor growth in bone via FABP4-dependent mechanisms. Oncotarget. 2013;4(11):2108-2123.

11. Gazi E, Gardner P, Lockyer NP, Hart CA, Brown MD, Clarke NW. Direct evidence of lipid translocation between adipocytes and prostate cancer cells with imaging FTIR microspectroscopy. J Lipid Res. 2007;48(8):1846-1856.

12. Liersch R, Muller-Tidow C, Berdel WE, Krug U. Prognostic factors for acute myeloid leukaemia in adults--biological significance and clinical use. Br J Haematol. 2014;165(1):17- 38

13. Feldman BJ, Streeper RS, Farese RV Jr, Yamamoto KR. Myostatin modulates adiogenesis to generate adipocytes with favorable metabolic effects. Proc Natl Acad Sci USA. 2006;103(42):15675-15680.

14. Bochet L, Lehuédé C, Dauvillier $\mathrm{S}$, et al. Adipocyte-derived fibroblasts promote tumor progression and contribute to the desmoplastic reaction in breast cancer. Cancer Res. 2013;73(18):5657-5668. 